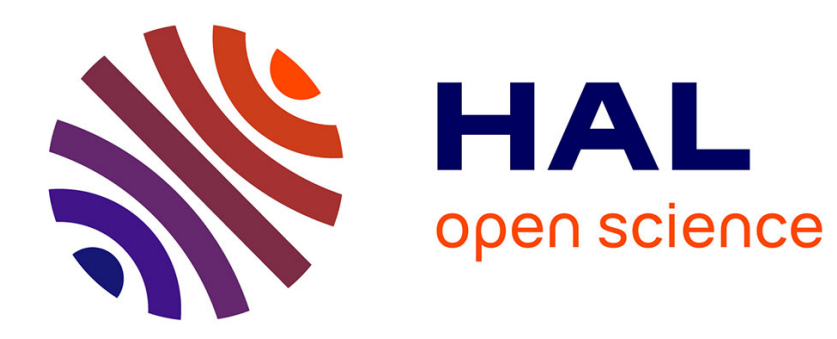

\title{
Algebras, automata and logic for languages of labeled birooted trees
}

\author{
David Janin
}

\section{To cite this version:}

David Janin. Algebras, automata and logic for languages of labeled birooted trees. ICALP, 2013, Riga, Latvia. pp.318-329, 10.1007/978-3-642-39212-2_29 . hal-00784898v2

\section{HAL Id: hal-00784898 \\ https://hal.science/hal-00784898v2}

Submitted on 25 Apr 2013

HAL is a multi-disciplinary open access archive for the deposit and dissemination of scientific research documents, whether they are published or not. The documents may come from teaching and research institutions in France or abroad, or from public or private research centers.
L'archive ouverte pluridisciplinaire HAL, est destinée au dépôt et à la diffusion de documents scientifiques de niveau recherche, publiés ou non, émanant des établissements d'enseignement et de recherche français ou étrangers, des laboratoires publics ou privés. 
Laboratoire Bordelais de Recherche en Informatique

Rapport de recherche RR-1467-13

\section{Algebras, automata and logic for languages of labeled birooted trees}

April 25, 2013

David Janin,

LaBRI, IPB, Université de Bordeaux 


\title{
Algebras, automata and logic for languages of labeled birooted trees
}

\author{
David Janin * \\ Université de Bordeaux, LaBRI UMR 5800, \\ 351, cours de la Libération, \\ F-33405 Talence, FRANCE \\ janin@labri.fr
}

\begin{abstract}
In this paper, we study the languages of labeled finite birooted trees: Munn's birooted trees extended with vertex labeling. We define a notion of finite state birooted tree automata that is shown to capture the class of languages that are upward closed w.r.t. the natural order and definable in Monadic Second Order Logic. Then, relying on the inverse monoid structure of labeled birooted trees, we derive a notion of recognizable languages by means of (adequate) premorphisms into finite (adequately) ordered monoids. This notion is shown to capture finite boolean combinations of languages as above. We also provide a simple encoding of finite (mono-rooted) labeled trees in an antichain of labeled birooted trees that shows that classical regular languages of finite (mono-rooted) trees are also recognized by such premorphisms and finite ordered monoids.
\end{abstract}

\section{Introduction}

Motivations and background. Semigroup theory has amply demonstrated its considerable efficiency over the years for the study and fine grain analysis of languages of finite words, that is subsets of the free monoid $A^{*}$. This can be illustrated most simply by the fact that a language $L \subseteq A^{*}$ is regular if and only if there is a finite monoid $S$ and a monoid morphism $\theta: A^{*} \rightarrow S$ such that $L=\theta^{-1}(\theta(L))$. In this case, we say that the language $L$ is recognized by the finite monoid $S$ (and the morphism $\theta$ ).

Even more effectively, for every language $L \subseteq A^{*}$, the notion of recognizability induces a notion of syntactic congruence $\simeq_{L}$ for the language $L$ in such a way that the monoid $M(L)=A^{*} / \simeq^{L}$ is the smallest monoid that recognizes $L$. Then, many structural properties of the language $L$ can be decided by analyzing the properties of its syntactic monoid $M(L)$, e.g. regularity, star freeness, etc (see [14] for more examples of such properties).

These results triggered the development of entire algebraic theories of languages of various structures elaborated on the basis of richer algebraic frameworks such as, among others, $\omega$-semigroups for languages of infinite words $[19$,

\footnotetext{
* Partially funded by project INEDIT, ANR-12-CORD-0009

Complete version of ICALP 2013 proceedings
} 
12], preclones or forest algebra for languages of trees $[5,3,2]$, or indeed $\omega$ hyperclones for languages of infinite trees [1]. With an aim to describing the more subtle properties of languages, several extensions of the notion of recognizability by monoids and morphisms were also taken into consideration, e.g. recognizability by monoids and relational morphisms [13] or recognizability by ordered monoids and monotonic morphisms [15].

A recent study of the languages of overlapping tiles $[6,9]$ or, equivalently, subsets of the (inverse) monoid of McAlister $[11,8]$, has led to the definition of quasi-recognizability: recognizability by means of (adequate) premorphisms into (adequately ordered) ordered monoids.

As (monotonic) morphisms are particular cases of premorphisms, this notion can be seen as a generalization of recognizability by (ordered) monoids and (monotonic) morphisms [15]. To some extent, quasi-recognizability can also be seen as a notion of co-algebraic recognizability in the sense that it is dual to the standard notion. Indeed, (adequate) premorphisms preserve some (and sufficiently many) decompositions while morphisms preserve all compositions.

However, this notion of quasi-recognizability has not yet been settled for we need to restrict both the class of allowed premorphisms and/or the class of finite ordered monoids for that notion to be effective. Without any restrictions, the inverse image by a premorphism of a finite subset of a finite ordered monoid may not even be computable [8]. Further still, there are several incomparable candidates for defining such an effective restriction as illustrated by a recent and complementary study of walking automata on birooted trees [7].

In this paper, we aim to stabilize the notion of recognizability by adequate premorphisms by applying it to the study of languages of labeled birooted trees. In doing so, it appears that this notion admits both simple automata theoretic characterization and robust logical characterization.

Outline. Birooted labeled trees, called birooted $F$-trees, are presented in Section 1. Equipped with an extension of Scheiblich's product of (unlabeled) birooted trees [16], the resulting algebraic structures are inverse monoids that are quite similar to discrete instances of Kellendonk's tiling semigroups [10]. Then, birooted $F$-trees can be ordered by the (inverse semigroup) natural order relation that is stable under product: the inverse monoid $\mathcal{B}^{1}(F)$ of labeled birooted $F$-trees is also a partially ordered monoid.

Birooted tree automata are defined and studied in Section 2. By construction, languages recognized by these finite automata are upward closed in the natural order. It follows that they fail to capture all languages definable by means of Monadic Second Order (MSO) formulae. However, this loss of expressive power is shown to be limited to the property of upward closure. Indeed, we prove (Theorem 2) that every upward closed language of birooted trees which is MSO definable is recognized by a finite state birooted tree automata.

As a case in point, when $F$ is seen as a functional signature, by embedding the classical $F$-terms (see [18]) into birooted $F$-trees, we show (Theorem 3 ) that the birooted tree image of every regular language $L$ of $F$-terms is of the form 
$U_{L} \cap D_{L}$ for some MSO definable and upward closed (resp. downward closed) language $U_{L}$ (resp. language $D_{L}$ ).

The algebraic counterpart of birooted tree automata is presented in Section 3 where the notions of adequately ordered monoids and adequate premorphisms are defined. The induced notion of quasi-recognizable languages of birooted $F$ trees is shown to be effective (Theorem 4).

As for expressive power, it is shown that every birooted tree automaton simply induces an adequate premorphism that recognizes the same language (Theorem 5) and that every quasi-recognizable language is MSO definable (Theorem 6). The picture is made complete by proving (Theorem 7) that quasirecognizable languages of birooted trees correspond exactly to finite boolean combinations of upward closed MSO definable languages.

Together with Theorem 3, this result demonstrates that our proposal can also be seen as yet another algebraic characterization of regular languages of trees that complete that previously obtained by means of preclones [5], forest algebras [3] or ordered monoids and admissible premorphisms [7].

Related works. We should also mention that the notion of birooted $F$-tree automata defined above is an extension of that previously defined [9] for languages of one-dimensional overlapping tiles: subsets of McAlister monoids [11].

Although closely related, we can observe that an extension of this type is by no means straightforward. Of course going from the linear structure of overlapping tiles to the tree shaped structure of birooted $F$-trees already induces a tangibly increased level of complexity. However, the main difference comes from edge directions. In overlapping tiles, all edges go in the same direction while, in birooted $F$-trees, edges can go back and forth (almost) arbitrarily. Proving Theorem 2 is thus much more complex than proving an analogous result for overlapping tiles.

Comparing our proposal with other known algebraic characterizations of languages of (mono-rooted) $F$-trees [5,3] is not easy. Of course, our proposal induces a larger class of definable languages since we are dealing with birooted $F$-trees and not just $F$-trees. However, a more relevant comparison would be to compare the classification of languages through a full series of approaches, by restricting even further the allowed recognizers: be them preclones as in [5], forest algebras [2] or adequately ordered monoids as proposed here.

With quasi-recognizability, recognizers are monoids (and premorphisms). It follows that the known restrictions applicable to the study of languages of words, e.g. aperiodic monoids [14], can simply be extended to adequately ordered monoids. Yet, the relevance of such restrictions for languages of mono-rooted or birooted $F$-trees still needs to be evaluated.

Another source of difficulty comes from the fact that adequate premorphisms are not morphisms : only disjoint products are preserved. To some extent, the notion of quasi-recognizability by premorphisms presented here is analogous, compared with classical recognizability by morphisms, to what unambiguous non deterministic automata are in comparison with deterministic automata. On the negative side, this means that the notion of quasi-recognizability has not yet 
been completely understood. On the positive side, this means that it may lead to radically new outcomes.

\section{Semigroups and monoids of birooted $F$-trees}

Simply said, a labeled birooted tree is a (non empty) finite connected subgraph of the Cayley graph of the free group $F G(A)$ with labeled vertices on some finite alphabet $F$ and two distinguished vertices respectively called the input root and the output root. This definition and some of the associated properties are detailled in this section.

\subsection{The free groups}

Formally, let $A$ be a finite (edge) alphabet and let $\bar{A}$ be a disjoint copy of $A$ with, for every letter $a \in A$, its copy $\bar{a} \in \bar{A}$. Let $u \mapsto \bar{u}$ be the mapping from $(A+\bar{A})^{*}$ to itself inductively defined by $\overline{1}=1$ and $\overline{u a}=\bar{a} \bar{u}$ and $\overline{u \bar{a}}=a \bar{u}$, for every $u \in(A+\bar{A})^{*}$, every $a \in A$. This mapping is involutive, i.e. $\overline{\bar{u}}=u$ for every $u \in(A+\bar{A})^{*}$, and it is an anti-morphism, i.e. $\overline{u v}=\bar{v} \bar{u}$ for every word $u$ and $v \in(A+\bar{A})^{*}$.

The free group $F G(A)$ generated by $A$ is the quotient of $(A+\bar{A})^{*}$ by the least congruence $\simeq$ such that, for every letter $a \in A, a \bar{a} \simeq 1$ and $\bar{a} a \simeq 1$. This is indeed a group since, for every $u \in(A+\bar{A})^{*}$, we have $[u][\bar{u}]=[1]$ hence $[\bar{u}]$ is the group inverse of $[u]$.

It is known that every class $[u] \in F G(A)$ contains a unique element $\operatorname{red}(u)$ (the reduced form of $u$ ) that contains no factors of the form $a \bar{a}$ nor $\bar{a} a$ for $a \in A$. In the sequel, every such class $[u] \in F G(A)$ is thus represented by its reduced form $\operatorname{red}(u)$. Doing so, the product $u \cdot v$ of every two reduced words $u$ and $v \in F G(A)$ is directly defined by $u \cdot v=\operatorname{red}(u v)$.

Elements of $F G(A)$, when seen as reduced words, can then be ordered by the prefix order relation $\leq_{p}$ defined, for every (reduced word) $u$ and $v \in F G(A)$ by $u \leq_{p} v$ when there exists (a reduced word) $w \in F G(A)$ such that $\operatorname{red}(u w)=$ $u w=v$. The associated predecessor relation $\prec_{p}$ is defined, for every $v$ and $w \in F G(A)$, by $v \prec_{p} w$ when $v<_{p} w$ and $w=v x$ for some $x \in A+\bar{A}$.

\subsection{Labeled birooted trees}

A labeled birooted tree on the edge alphabet $A$ and the vertex alphabet $F$ is a pair $B=\langle t, u\rangle$ where $t: F G(A) \rightarrow F$ is a partial maps which domain $\operatorname{dom}(t)$ is a prefix closed subset of $F G(A)$ with $u \in \operatorname{dom}(t)$. In such a presentation, $1 \in \operatorname{dom}(t)$ is the input root vertex and $u \in \operatorname{dom}(t)$ is the output root vertex. Assuming the edge alphabet $A$ is implicit, these labeled birooted trees are called birooted $F$-trees or, when $F$ is also implicit, simply birooted trees.

For every birooted tree $B=\langle t, u\rangle$, for every $v \in \operatorname{dom}(t)$, let $t_{v}: F G(A) \rightarrow F$ be the partial function defined by $\operatorname{dom}\left(t_{v}\right)=\bar{v} \cdot \operatorname{dom}(t)$ and $t_{v}(w)=t(v w)$ for 
every $w \in \operatorname{dom}\left(t_{v}\right)$. Accordingly, let $B_{v}=\left\langle t_{v}, \bar{v} u\right\rangle$ be the $v$ translation of the birooted tree $B$.

Observe that such a translation slightly differs from the classical notion of subtrees since $\operatorname{dom}\left(t_{v}\right)=\bar{v} \cdot \operatorname{dom}(t)$ contains as many vertices as $\operatorname{dom}(t)$. A notion of sub-birooted tree $B_{v}^{p}$, with fewer vertices and thus more closely related with the classical notion of subtree, is defined below when proving the decomposition property (Lemma 1).

The partial product $\langle r, u\rangle \cdot\langle s, v\rangle$ of two birooted $F$-tree $\langle r, u\rangle$ and $\langle s, v\rangle$ is defined, when it exists, as the birooted $F$-tree $\langle t, w\rangle$ defined by $w=u \cdot v$, $\operatorname{dom}(t)=\operatorname{dom}(r) \cup u \cdot \operatorname{dom}(s), t\left(u^{\prime}\right)=r\left(u^{\prime}\right)$ for every $u^{\prime} \in \operatorname{dom}(r)$ and $t_{u}\left(v^{\prime}\right)=$ $s\left(v^{\prime}\right)$ for every $v^{\prime} \in \operatorname{dom}(s)$.

Observe that such a product exists if and only if the tree $r_{u}$ and the tree $s$ agree on $\operatorname{dom}\left(r_{u}\right) \cap \operatorname{dom}(s)$, i.e. for every $v^{\prime} \in \operatorname{dom}\left(r_{u}\right) \cap \operatorname{dom}(s)$, we have $r_{u}\left(v^{\prime}\right)=r\left(u v^{\prime}\right)=s\left(v^{\prime}\right)$. It follows that undefined products may arise when $F$ is not a singleton.

Two examples of birooted $F$-trees $B_{1}$ and $B_{2}$ are depicted in Figure 1 , with a dangling input edge marking the input root and a dangling output edge marking the output root.
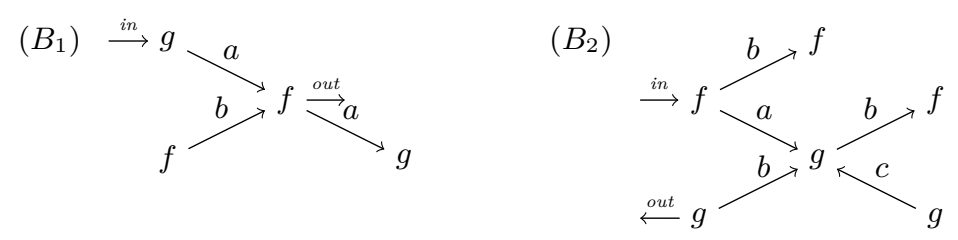

Fig. 1. Two birooted $F$-trees $B_{1}$ and $B_{2}$

The (defined) product of the birooted $F$-trees $B_{1}$ and $B_{2}$ is then depicted in Figure 2 . In that picture, the cercle marks the synchronization vertex that

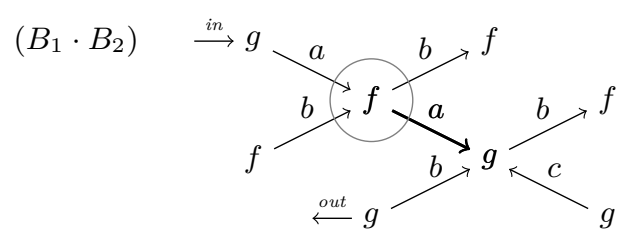

Fig. 2. The product $B_{1} \cdot B_{2}$ of the two birooted $F$-trees $B_{1}$ and $B_{2}$

results from the merging of the output root of $B_{1}$ and the input root of $B_{2}$. The 
$a$-labeled edge $f \stackrel{a}{\rightarrow} g$ emanating from that vertex is the common edge resulting from the fusion of the two (synchronized) birooted $F$-trees.

The product is completed by adding a zero element for the undefined case with $0 \cdot\langle t, v\rangle=\langle t, v\rangle \cdot 0=0 \cdot 0=0$ for every (defined) birooted tree $\langle t, v\rangle$.

One can easily check that the completed product is associative. The resulting structure is thus a semigroup denoted by $\mathcal{B}(F)$ : the semigroup of birooted $F$ trees. When $F$ is a singleton, every birooted $F$-tree can just be seen as a pair $(P, u)$ with an non empty prefix closed domain $P \subseteq F G(A)$ and an output root $u \in P$. Then, following Scheiblich presentation [16], the semigroup $\mathcal{B}(F)$ is the free monoid $\operatorname{FIM}(A)$ generated by $A$ with unit $1=(\{1\}, 1)$. When $F$ is not a singleton, we extend the set $\mathcal{B}(F)$ with a unit denoted by 1 . The resulting structure is a monoid denoted by $\mathcal{B}^{1}(F)$ : the monoid of birooted $F$-trees.

The monoid of birooted $F$-trees is an inverse monoid, i.e. for every $B \in \mathcal{B}^{1}(F)$ there is a unique $B^{-1} \in \mathcal{B}^{1}(F)$ such that $B B^{-1} B=B$ and $B^{-1} B B^{-1}=B^{-1}$. Indeed, we necessarily have $0^{-1}=0,1^{-1}=1$ and, for every non trivial birooted $F$-tree $\langle t, u\rangle$ one can check that $\langle t, u\rangle^{-1}=\left\langle t_{u}, \bar{u}\right\rangle$.

As an inverse monoid, elements of $\mathcal{B}^{1}(F)$ can be ordered by the natural order defined, for every $B$ and $C \in \mathcal{B}^{1}(F)$ by $B \leq C$ when $B=B B^{-1} C$ (equivalently $B=C B^{-1} B$ ). One can check that 0 is the least element and, for every defined birooted $F$-trees $\langle r, u\rangle$ and $\langle s, v\rangle$ we have $\langle r, u\rangle \leq\langle s, v\rangle$ if and only if $u=v$, $\operatorname{dom}(r) \supseteq \operatorname{dom}(s)$ and, for every $w \in \operatorname{dom}(s), t(w)=s(w)$.

Observe that, as far as trees only are concerned, the natural order is the reverse of the (often called) prefix order on trees. In particular, the bigger is the size of a birooted tree, the smaller is the birooted tree in the natural order.

\subsection{Strong decomposition of birooted trees}

One can easily check that the monoid of birooted $F$-trees is finitely generated. We prove here a stronger statement that will be extensively used in the remainder of the text.

A birooted tree is said elementary when it is either 0 or 1 , or of the from $B_{f}=\langle\{1 \mapsto f\}, 1\rangle$ for some $f \in F$ or of the form $B_{f x g}=\langle\{1 \mapsto f, x \mapsto g\}, x\rangle$ for some vertex label $f$ and $g \in F$ and some letter $x \in A+\bar{A}$.

$$
\left(B_{f a g}\right) \stackrel{\text { in }}{\longrightarrow} f \stackrel{a}{\longrightarrow} g \stackrel{\text { out }}{\longrightarrow} \quad\left(B_{f}\right) \stackrel{\text { in }}{\longrightarrow} f \stackrel{\text { out }}{\longrightarrow} \quad\left(B_{f \bar{a} g}\right) \stackrel{\text { in }}{\longrightarrow} f \stackrel{a}{ } g \stackrel{\text { out }}{\longrightarrow}
$$

Fig. 3. The elementary birooted $F$-trees $B_{f a g}, B_{f}$ and $B_{f \bar{a} g}$

The right projection $B^{R}$ (resp. the left projection $B^{L}$ ) of a birooted tree $B=\langle t, u\rangle$ is defined to be $B^{R}=B B^{-1}$ (resp. $B^{L}=B^{-1} B$ ) or, equivalently, $B^{R}=\langle t, 1\rangle\left(\right.$ resp. $\left.B^{L}=\left\langle t_{u}, 1\right\rangle\right)$. The right projection $B^{R}$ of $B$ is also called the reset of $B$. 
The product $B_{1} \cdot B_{2}$ of two birooted trees $B_{1}$ and $B_{2}$ is a disjoint product when $B_{1} \cdot B_{2} \neq 0$ and there is a unique elementary birooted tree $B_{f}$ such that $B_{1}^{L} \leq B_{f}$ and $B_{2}^{R} \leq 1$, i.e. $B_{1}^{L} \vee B_{2}^{R}=1$. This restricted product is called a disjoint product because, when $B_{1}=\left\langle t_{1}, u_{1}\right\rangle$ and $B_{2}=\left\langle t_{2}, u_{2}\right\rangle$, the product $B_{1} \cdot B_{2}$ is disjoint if and only if $t\left(u_{1}\right)=t_{2}(1)=f$ and $\operatorname{dom}\left(t_{1}\right) \cap u_{1} \cdot \operatorname{dom}\left(t_{2}\right)=\left\{u_{1}\right\}$, i.e. the set of edges in $B_{1} \cdot B_{2}$ is the disjoint union of the set of edges of $B_{1}$ and the set of (translated) edges of $B_{2}$.

Lemma 1 (Strong decomposition). For every $B \in \mathcal{B}(F)$, the birooted $F$-tree $B$ can be decomposed into a finite combination of elementary birooted trees by disjoint products and (right) resets.

Proof. Let $B=\langle t, u\rangle$ be a birooted $F$-tree. We aim at proving it can be decomposed as stated above. We first define some specific sub-birooted trees of $B$ that will be used for such a decomposition.

For every vertex $v$ and $w \in \operatorname{dom}(t)$ such that $v \prec_{p} w$, let $B_{v, w}^{p}$ be the two vertices birooted $F$-tree defined by $B_{v, w}^{p}=B_{f x g}$ where $f=t(v), g=t(w)$ and $v x=w$.

Let $U=\left\{v \in \operatorname{dom}(t): 1 \leq_{p} v \leq_{p} u\right\}$ be the set of vertices that appears on the path from the input root 1 to the output root $u$.

For every $v \in \operatorname{dom}(t)$, let $D^{p}(v)$ be the greatest prefix closed subset of the set $\left\{w \in \operatorname{dom}\left(t_{v}\right): v \leq_{p} v w, v w \in U \Rightarrow w=1\right\}$ and let $B_{v}^{p}=\left\langle t_{v} \mid D^{p}(v), 1\right\rangle$ be the idempotent birooted tree obtained from $B$ by restricting the subtree $t_{v}$ rooted at the vertex $v$ to the domain $D^{p}(v)$.

These basic ingredients of the expected decomposition are depicted Figure 4 below.

Then, given $u_{0}=1<_{p} u_{1}<_{p} u_{2}<_{p} \cdots<_{p} u_{n-1}<_{p} u_{n}=u$ the increasing sequence (under the prefix order) of all the prefixes of the output root $u$, we observe that $B=B_{u_{0}}^{p} B_{u_{0}, u_{1}}^{p} B_{u_{1}}^{p} \cdots B_{u_{n-1}}^{p} B_{u_{n-1}, u_{n}}^{p} B_{u_{n}}^{p}$ with only disjoint products.

It remains thus to prove that every idempotent sub-birooted tree of the form $B_{v}^{p}$ for some $v \in \operatorname{dom}(t)$ can also be decomposed into an expression of the desired form. But this is easily done by induction on the size of the birooted trees $B_{c}^{p}$.

Indeed, Let $v \in \operatorname{dom}(t)$. In the case $v$ is a leaf (w.r.t. the prefix order) then $B_{v}^{p}=B_{t(v)}$ and we are done. Otherwise, we have $B_{v}^{p}=\langle r, 1\rangle$ for some $F$-tree $r$ and we observe that $B_{v}^{p}=\prod\left\{\left(B_{v, w}^{p} \cdot B_{w}^{p}\right)^{R}: w \in \operatorname{dom}(r), v \prec_{p} w\right\}$ with only disjoint products and resets. This concludes the proof.

The above decomposition of $B$ as a combination of elementary birooted trees by disjoint products and right projections is called a strong decomposition of the birooted $F$-tree $B$.

Example. The basic elements involved in a strong decomposition, be them one edge birooted trees of the form $B_{v, w}^{p}$ or idempotent birooted trees of the form $B_{v}^{p}$, are depicted in the example below.

The birooted tree $B$ has trivial vertex labeling, i.e. $F$ is a singleton. However, for convenience, every vertex is labeled by its corresponding path from the input 
root. Also, the edges on the path from the input root to the output root (that go through all vertices of $U$ in the proof above) are marked by dotted arrows.

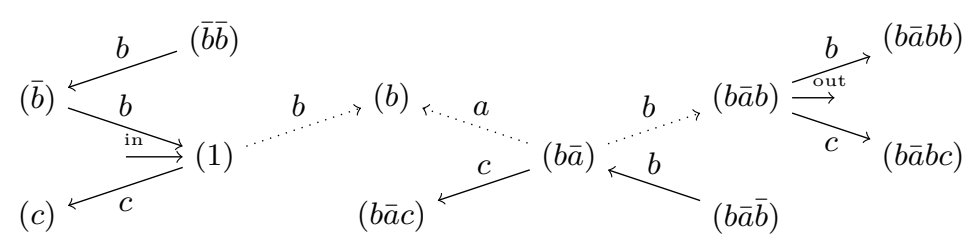

$\left(B_{1}^{p}\right)$

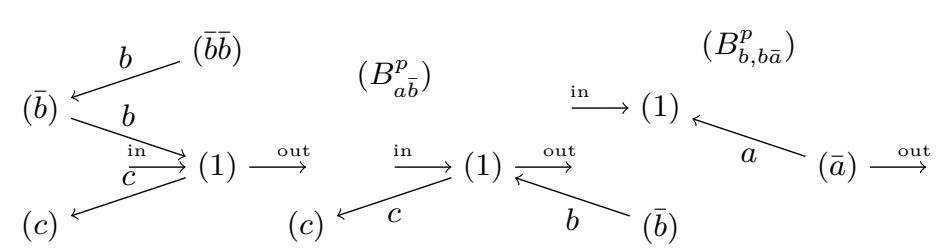

Fig. 4. A birooted tree $B$ and some elements of its decomposition

Here, the decomposition starts with $B=B_{1}^{p} \cdot B_{1, b} \cdot B_{b}^{p} \cdot B_{b \bar{a}}^{p} \cdot B_{b \bar{a}, b \bar{a} b}^{p} \cdot B_{b \bar{a} b}^{p}$.

\section{Birooted $\boldsymbol{F}$-tree automata}

In this section, we define the notion of birooted $F$-tree automata that is shown to capture the class of languages of birooted $F$-trees that are upward closed w.r.t. the natural order and definable in Monadic Second Order Logic (MSO).

\subsection{Definition}

A birooted $F$-tree (finite) automaton is a quintuple $\mathcal{A}=\langle Q, \delta, \Delta, W\rangle$ defined by a (finite) set of states $Q$, a (non deterministic) state table $\delta: F \rightarrow \mathcal{P}(Q)$, a (non deterministic) transition table $\Delta: A \rightarrow \mathcal{P}(Q \times Q)$ and an acceptance condition $W \subseteq Q \times Q$.

A run of the automaton $\mathcal{A}$ on a non trivial birooted $F$-tree $B=\langle t, u\rangle$ is a mapping $\rho: \operatorname{dom}(t) \rightarrow Q$ such that for every $v \in \operatorname{dom}(t)$ :

$\triangleright$ State coherence: $\rho(v) \in \delta(t(v))$,

$\triangleright$ Transition coherence: for every $a \in A$, if $v a \in \operatorname{dom}(t)$ then $(\rho(v), \rho(v a)) \in$ $\Delta(a)$ and if $v \bar{a} \in \operatorname{dom}(t)$ then $(\rho(v \bar{a}), \rho(v)) \in \Delta(a)$.

The run $\rho$ is an accepting run when $(\rho(1), \rho(u)) \in W$. The set $L(\mathcal{A}) \subseteq \mathcal{B}(F)$ of birooted $F$-tree $B$ such that there is an accepting run of $\mathcal{A}$ on $B$ is the language recognized by the automaton $\mathcal{A}$. 


\subsection{An automaton example}

Assume that $A=\{a, b, c\}$. Assume also that $F=\{0\}$. Since $F$ is a singleton, this means that $\mathcal{B}(F)$ is (isomorphic to) the free inverse monoid $F I M(A)$ generated by $A$.

Let then $\mathcal{A}=\langle Q, \delta, \Delta, W\rangle$ be the birooted tree automaton defined by:

$\triangleright Q=(\star, \star)+\{1, a, \bar{a}, b, \bar{b}\} \times\{1, a, \bar{a}, b, \bar{b}\}$,

$\triangleright \delta(\circ)=Q$,

$\triangleright \Delta(x)$ defined by all transitions of one the following form:

- $(\star, \star) \stackrel{x}{\longrightarrow}(y, z)$ when $y \neq \bar{x}$ and $z \neq \bar{x}$,

- $(\star, \star) \stackrel{x}{\longleftarrow}(y, z)$ when $y \neq x$ and $z \neq x$,

- $(y, x) \stackrel{x}{\longrightarrow}(\bar{x}, z)$ when $y \neq x$ and $z \neq \bar{x}$,

- $(y, \bar{x}) \stackrel{x}{\longleftarrow}(x, z)$ when $y \neq \bar{x}$ and $z \neq x$,

$\triangleright W=(\{1\} \times\{1, a, b, \bar{a}, \bar{b}\}) \times(\{1, a, b, \bar{a}, \bar{b}\} \times\{1\})$

A typical accepting run of the automaton $\mathcal{A}$ on a birooted tree is depicted in Figure 5 below.

Again, edges from the input root to the output root are dotted and vertices are marked by the states of the run.

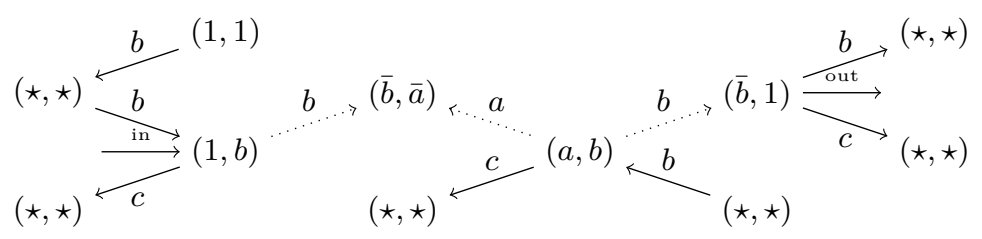

Fig. 5. A root path detector automaton

One can observe, in an accepting run, the automaton $\mathcal{A}$ necessarily and uniquely identifies the path from the input root to the output root, by labeling every vertices on that path by a state of the form $(x, y) \in A^{\prime} \times A^{\prime}$ with $A^{\prime}=$ $\{1, a, \bar{a}, b, \bar{b}\}$ with $x$ that tells what is the (directed) edge towards the input root, and $y$ that tells what is the (directed) edge towards the output root. In particular, the input root is labeled by a state of the form $(1, y)$ and the output root is labeled by a state of the form $(x, 1)$. Moreover, since neither $c$ nor $\bar{c}$ belongs to $A^{\prime}$, this prevents $c$ to appear on that path.

Of course, states of the form $(x, y) \in A^{\prime} \times A^{\prime}$ may also occur out of the path from the input to the output, drawing disconnected paths on the birooted tree. The vertex at position $\bar{b} \bar{b}$ from the input root is such an example. However, since these path are necessarily disconnected, they cannot be confused by the path between the roots. 
One can easily adapt this example to define other automata that only accept idempotent birooted trees, or positive birooted trees (with edges between the roots all oriented forward: from the input to the output), or negative trees birooted trees (with edges between the roots all oriented backward: from the output to the input), or even just directed trees (with at most one input edge per vertices) etc...

\subsection{Expressive power}

Every non trivial birooted $F$-tree $B=\langle t, u\rangle$ can be seen as a (tree-shaped) FO-structure $\mathcal{M}_{B}$ with domain $\operatorname{dom}\left(\mathcal{M}_{B}\right)=\operatorname{dom}(t)$, constant $i n_{B}=1$ and constant out $_{B}=u$, unary relation $S_{f}=t^{-1}(f)$ for every $f \in F$ and binary relation $R_{a}=\{(v, w) \in \operatorname{dom}(t) \times \operatorname{dom}(t): v a=w\}$ for every $a \in A$.

We say that a language $L \subseteq \mathcal{B}(F)$ is definable in monadic second order logic (MSO) when there exists a closed MSO formula $\varphi$ on the FO-signature $\{$ in, out $\} \cup\left\{S_{f}\right\}_{f \in F} \cup\left\{R_{a}\right\}_{a \in A}$ such that $L=\left\{B \in \mathcal{B}(F): \mathcal{M}_{B} \models \varphi\right\}$.

The following theorem gives a rather strong characterization of the languages recognized by finite state birooted $F$-tree automata.

Theorem 2. Let $L \subseteq \mathcal{B}(F)$ be a language of birooted $F$-trees. The language is recognized by a finite birooted $F$-tree automaton if and only if $L$ is upward closed (in the natural order) and MSO definable.

Proof. Let $L \subseteq \mathcal{B}(F)$ be a language of birooted $F$-trees. We first prove the easiest direction, from birooted tree automata to MSO. Then, we prove the slightly more difficult direction from MSO to birooted tree automata.

From birooted tree automata to $M S O$. Assume that $L$ is recognizable by a finite state birooted tree automaton $\mathcal{A}$. Without loss of generality, since $\mathcal{A}$ is finite, we assume that the set $Q$ of states of $\mathcal{A}$ is such that $Q \subseteq \mathcal{P}([1, n])$ for some $n \geq \log _{2}|Q|$.

Then, checking that a birooted tree $\langle t, u\rangle$ belongs to $L(\mathcal{A})$ just amounts to checking that there exists an accepting run. This can easily be described by an existential formula of monadic second order logic of the form $\exists X_{1} X_{2} \cdots X_{n} \varphi($ in, out $)$ with $n$ set variables $X_{1}, X_{2}, \ldots, X_{n}$ and a first order formula $\varphi($ in, out $)$.

Indeed, every mapping $\rho: \operatorname{dom}(v) \rightarrow Q$ is encoded by saying, for every vertex $v \in \operatorname{dom}(t)$, that $\rho(v)=\left\{k \in[1, n]: v \in X_{k}\right\}$. Then, checking that the mapping $\rho$ encoded in such a way is indeed an accepting run amounts to checking that it satisfies state and transition coherence conditions and acceptance condition. This is easily encoded in the FO-formula $\varphi(x, y)$.

From MSO to birooted tree automata. Conversely, assume that $L$ is upward closed for the natural order and that $L$ is definable in $M S O$. Observe that every $B=\langle t, u\rangle$ can just be seen as a (deterministic) tree rooted in the input root vertex 1 with edges labeled on the alphabet $A+\bar{A}$ (with edge "direction" being induced by the prefix order on $F G(A)$ ), vertices labeled on the alphabet 
$F \times\{0,1\}$ (with 1 used to distinguish the output root $u$ from the other vertices) An example of such an encoding of birooted trees into trees is depicted in Figure 6
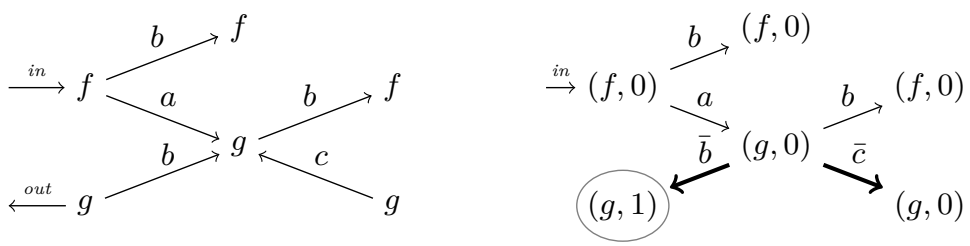

Fig. 6. From a birooted $F$-tree to a mono-rooted tree with $(A+\bar{A})$-labeled edges

Since $L$ is definable in MSO, applying (an adapted version of) the theorem of Doner, Thatcher and Wright (see for instance [18]), there exists a finite state tree automaton $\mathcal{A}$ that recognizes $L$. We conclude our proof by defining from the (finite) tree automaton $\mathcal{A}$ a (finite) birooted tree automaton $\mathcal{A}^{\prime}$ such that $L(\mathcal{A})=L\left(\mathcal{A}^{\prime}\right)$.

The major difficulty in defining $\mathcal{A}^{\prime}$ is that the (one root) tree automaton $\mathcal{A}$ reads a tree from the (input) root to the leaves hence following the prefix relation order $\leq_{p}$. Moreover, birooted trees, such a prefix order is not encoded in the direction of edges. It follows that, when translating the tree automaton $\mathcal{A}$ into an equivalent birooted tree automaton $\mathcal{A}^{\prime}$, we need to encode (and propagate) that direction information into states.

But this can be achieved by observing that for every vertex $v$ and $w$ such that $v \prec_{p} w$, the edge from $v$ to $w$ is uniquely defined by the letter $x \in(A+\bar{A})$ such that $v x=w$. It follows that every such a vertex $w$ (distinct from the input root 1) will be marked in automaton $\mathcal{A}^{\prime}$ by a state that will encode that letter $x$; distinguishing thus the unique predecessor vertex $v$ of $w$ from all successor vertices $w^{\prime}$ such that $w \prec_{p} w^{\prime}$.

The details of the proof arguments are given below.

The (mono-rooted) tree automaton $\mathcal{A}$ recognizing $L$. Let $\mathcal{A}$ be a non deterministic top down tree automaton on the edge alphabet $A+\bar{A}$ that recognizes $L$.

Adapting classical definitions to birooted trees seen as trees, we can define $\mathcal{A}$ by $\mathcal{A}=\left\langle Q, I_{0}, \delta, T\right\rangle$ with a finite set of states $Q$, an set of initial states $I \subseteq Q$ (though a priori reducible to a single initial state, this possibility is used below for normalizing $\mathcal{A}$ ), a (non deterministic) transition function $\delta: Q \times F \times\{0,1\} \rightarrow$ $\mathcal{P}(\mathcal{P}((A+\bar{A}) \times Q))$ and a specification of accepting states $T: F \rightarrow \mathcal{P}(Q)$ that recognized the birooted tree language $L$ with birooted trees interpreted as trees as described above.

With these notations, an accepting run of automaton $\mathcal{A}$ on a birooted $F$-tree $B=\langle t, u\rangle$ (seen as a tree rooted in 1 ) is a mapping $\rho: \operatorname{dom}(t) \rightarrow Q$ such that $\rho(1) \in I_{0}$ and, for every vertex $v \in \operatorname{dom}(t)$ : 
$\triangleright$ inner vertex condition: if $v$ is non maximal (in the prefix order) then the set of pairs $\left\{(x, \rho(v x)) \in(A+\bar{A}) \times Q: x \in(A+\bar{A}), v<_{p} v x, v x \in \operatorname{dom}(t)\right\}$ either belongs to $\delta(\rho(v), t(v), 0)$ when $u \neq v$ or belongs to $\delta(\rho(v), t(v), 1)$ when $u=v$,

$\triangleright$ leaf vertex condition: if $v$ is maximal (in the prefix order) then $\rho(v) \in T(t(v)$ ).

The language of birooted trees $L(\mathcal{A}) \subseteq \mathcal{B}(\mathcal{A})$ recognized by $\mathcal{A}$ is defined as the set of birooted trees for which there exists an accepting run of $\mathcal{A}$ on $B$. By assumption, we have $L=L(\mathcal{A})$.

We observe that, for every $p \in Q$, every $f \in F$ and every $m \in\{0,1\}$, the following assumptions can be done on $\delta(p, f, m)$ without altering the language $L(\mathcal{A})$.

Since all the trees are deterministic in the edge alphabet $A+\bar{A}$ we can assume that for every $X \in \delta(p, f, m)$ and $x \in A+\bar{A}$, there exists at most one state $q \in Q$ such that $(x, q) \in X$, i.e. $X$ models a partial function from $A+\bar{A}$ to $Q$.

Since we are only interested in accepting runs, we can also assume that we have $\emptyset \in \delta(p, f, x)$ if and only if $p \in T(f)$. This means that the inner vertex condition and the leaf vertex condition can be merged into a single condition : the inner vertex condition extended to all vertices.

Since $L$ is upward closed w.r.t. the natural order, we may also assume that $\delta(p, f, m) \subseteq \mathcal{P}((A+\bar{A}) \times Q)$ is closed under taking non empty subsets, i.e. for every set non empty $X \in \mathcal{P}((A+\bar{A}) \times Q)$, if there is $Y \in \delta(p, f, m)$ such that $X \subseteq Y$ then $X \in \delta(p, f, m)$.

A little harder, we can also assume that $Q=Q_{0} \uplus Q_{1} \uplus Q_{2}$ (with $\uplus$ standing for the disjoint union) such that $I_{0} \subseteq Q_{0} \uplus Q_{1}$ and for every for every $p \in Q$, every $f \in F$ :

$\triangleright$ if $p \in Q_{0}$ then $\delta(p, f, 1)=\emptyset$ (henceforth such a transition is unrealizable) and, for every $X \in \delta(p, f, 0)$ there exists one and only one pair $(x, q) \in X$ such that $q \in Q_{0} \cup Q_{1}$, and, for every other pair $\left(y, q^{\prime}\right) \in X$ we have $q^{\prime} \in Q_{2}$, $\triangleright$ if $p \in Q_{1}$ then $\delta(p, f, 0)=\emptyset$ (idem) and $\delta(p, f, 1) \subseteq \mathcal{P}\left((A+\bar{A}) \times Q_{2}\right.$, $\triangleright$ if $p \in Q_{2}$ then $\delta(p, f, 1)=\emptyset$ (idem) and $\delta(p, f, 0) \subseteq \mathcal{P}\left((A+\bar{A}) \times Q_{2}\right.$.

Indeed, possibly taking $Q \times\{0,1,2\}$ with $Q_{0}=Q \times\{0\}, Q_{1}=Q \times\{1\}$ and $Q_{2}=$ $Q \times Q$ as new set of states with $I_{0} \times\{0,1\}$ as new set of initial states, this just amounts to extending the transition function in such a (quite straightforward) way that, for every accepting run $\rho: \operatorname{dom}(t) \rightarrow Q$ on a birooted tree $B=\langle t, u\rangle$, for every $v \in \operatorname{dom}(t)$, if $v<_{p} u$ then $\rho(v) \in Q_{0}$, if $v=u$ then $\rho(v) \in Q_{1}$ and $\rho(v) \in Q_{2}$ in all other cases.

In other words, the states of $Q_{0}$ can only be used on the vertices encountered along the (shortest) path from the input root (included when distinct from the output root) to the output root (excluded), states of $Q_{1}$ can only be used on the output root, and states of $Q_{2}$ can only be used on all other vertices. This situation is depicted in the Figure 7 below.

It follows that the definition of automaton $\mathcal{A}$ can be simplified into $\mathcal{A}=$ $\left\langle Q, Q_{1}, I_{0}, \delta\right\rangle$ with set of states $Q=Q_{0} \uplus Q_{1} \uplus Q_{2}$ as above, initial states $I_{0} \subseteq$ 


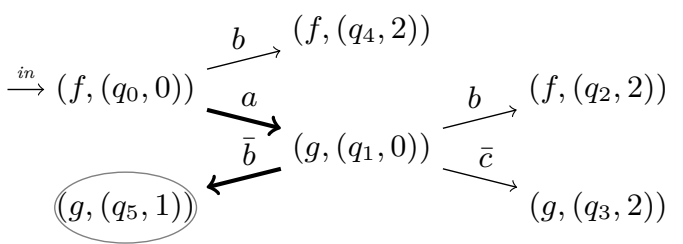

Fig. 7. A run of $\mathcal{A}$ on a tree $(A+\bar{A})$-labeled edges

$\mathcal{P}\left(Q_{0} \uplus Q_{1}\right)$, transition table $\delta: Q \times F \rightarrow \mathcal{P}(\mathcal{P}((A+\bar{A}) \times Q))$ restricted as above, with the additional condition that, in accepting runs, the output root must be labeled by a state of $Q_{1}$.

We are now ready to simulate the tree automaton $\mathcal{A}$, with the distinguished set of state $Q_{1}$ for the output root by a birooted tree automaton $\mathcal{A}^{\prime}$.

The birooted tree automaton $\mathcal{A}^{\prime}$ simulating $\mathcal{A}$. Let $\mathcal{A}^{\prime}=\left\langle Q^{\prime}, \delta^{\prime}, \Delta^{\prime}, W^{\prime}\right\rangle$ be the birooted tree automaton defined as follows:

$\triangleright$ the set of states $Q^{\prime} \subseteq(1+A+\bar{A}) \times Q \times F \times \mathcal{P}((A+\bar{A}) \times Q)$ is defined as the set of triples of the form $(x, p, f, X)$ such that any of the following condition is satisfied:

- input root vertex states: $x=1, p \in I_{0}$ and $X \in \delta(p, f)$,

- inner vertex states: $x \neq 1$ and $X \neq \emptyset$ with $X \in \delta(p, f)$.

- leaf vertex state: no condition on $x$ (since the root can be a leaf), $p \in T$ and $X=\emptyset$ with $X \in \delta(p, f)$.

$\triangleright$ the state table $\delta^{\prime}: F \rightarrow Q^{\prime}$ is defined, for every $f \in F$ by $\delta^{\prime}(f) \subseteq Q^{\prime}$ is the set of states of $\mathcal{A}^{\prime}$ that are of the form $(x, p, f, X)$,

$\triangleright$ the transition table $\Delta^{\prime}: A \rightarrow \mathcal{P}\left(Q^{\prime} \times Q^{\prime}\right)$ is defined, for every $a \in A$ by $\Delta^{\prime}(a) \subseteq Q^{\prime} \times Q^{\prime}$ is the set of pairs states of the form $((x, p, f, X),(y, q, g, Y))$ such that:

- either $y=a$ and $(a, q) \in X$ (and $X \in \delta(p, f)$ by definition of states),

- or $x=\bar{a}$ and $(\bar{a}, p) \in Y$ (and $Y \in \delta(q, f)$ by definition of states),

$\triangleright$ the acceptance condition $W^{\prime}$ is defined as the set of states of $\mathcal{A}^{\prime}$ of the form $((1, p, X),(y, q, Z))$ in $Q^{\prime} \times Q^{\prime}$ such that $p \in I_{0}$ and $q \in Q_{1}$.

We conclude the proof by showing that $L\left(\mathcal{A}^{\prime}\right)=L(\mathcal{A})$. In order do so, let $B=$ $\langle t, u\rangle$ be a birooted $F$-tree.

From a tree run to a birooted tree run. Let $\rho: \operatorname{dom}(t) \rightarrow Q$ be an accepting run of automaton $\mathcal{A}$ on the birooted tree $B$ (hence with $\rho(u) \in Q_{1}$ ). We define $\rho^{\prime}: \operatorname{dom}(t) \rightarrow Q^{\prime}$ by taking, for every $v \in \operatorname{dom}(t)$, the state $\rho^{\prime}(v)=$ $(x, \rho(v), t(v), X) \in Q^{\prime}$ with:

$\triangleright$ if $v=1$ then $x=1$ and if $v \neq 1$ then $x \in A+\bar{A}$ with $v=w x$ for some $w<{ }_{p} v$, 
$\triangleright X=\left\{(x, p) \in(A+\bar{A}) \times Q: v x \in \operatorname{dom}(t), v<_{p} v x, p=\rho(v x)\right\}$.

By definition of $\mathcal{A}^{\prime}$, the mapping $\rho^{\prime}$ built from $\rho$ as above is indeed an accepting run of $\mathcal{A}^{\prime}$ on $B$.

From birooted tree run to tree run. Conversely, let $\rho^{\prime}: \operatorname{dom}(t) \rightarrow Q^{\prime}$ be an accepting run of automaton $\mathcal{A}^{\prime}$ on $B$. We define $\rho: \operatorname{dom}(t) \rightarrow Q$ by taking, for every $v \in \operatorname{dom}(t), \rho(v)=p$ when $\rho^{\prime}(v)$ is of the form $(x, p, f, X)$.

The following fact is easily proved by induction on the distance of vertices from the root. For every $v \in d o m(t)$ :

$\triangleright$ if the state $\rho^{\prime}(v)$ is of the form $(1, p, f, X)$ then $v=1, p=\rho(v), t(v)=f$, and $X \in \delta(p, f)$,

$\triangleright$ if the state $\rho^{\prime}(v)$ is of the form $(x, p, f, X)$ with $x \in A+\bar{A}$, then, given $w=v x$ (in $F G(A)$ ) we have $w<_{p} v=w \bar{x}, p=\rho(v), f=t(v)$ and, given $\alpha(t, x)=\{y \in A+\bar{A}: y \neq x, v y \in \operatorname{dom}(t)\}$, for every $y \in B, v \leq_{p} v y$ and $X=\{(y, \rho(v y)) \in(A+\bar{A}) \times Q: y \in \alpha(t, x)\}$ with $X \in \delta(\rho(v), f)$.

These properties explicit how indeed the automaton $\mathcal{A}^{\prime}$ in the accepting run $\rho^{\prime}$ marks the shortest path from every vertex to the (input) root. These properties also prove that the mapping $\rho$ defined from $\rho^{\prime}$ is indeed an accepting run of $\mathcal{A}$ on the birooted tree $B$.

From now on, a language of birooted $F$-trees that is definable by a finite birooted $F$-tree automaton is called a regular language of birooted $F$-trees.

\subsection{Tree languages vs birooted tree languages}

We aim now at relating languages of birooted $F$-trees and languages of $F$-trees. Assume till the end of that section that the set $F$ is now a finite functional signatures that is a finite set of symbols equipped with some arity mapping $\rho: F \rightarrow \mathcal{P}(A)$ that maps every function symbol $f$ the set of its arguments' names $\rho(f) \subseteq A$.

A $F$-tree (also called $F$-term) is a function $t: A^{*} \rightarrow F$ with prefix closed finite domain $\operatorname{dom}(t)$ such that for every $u \in \operatorname{dom}(t)$, every $a \in A$, if $u a \in \operatorname{dom}(t)$ then $a \in \rho(t(u))$. Such a finite tree $t$ is said to be complete when, moreover, for every $u \in \operatorname{dom}(t)$, for every $a \in A$, if $a \in \rho(t(u))$ then $u a \in \operatorname{dom}(t)$.

Every $F$-tree $t$ is encoded into a birooted $F$-tree $\langle t, 1\rangle$ called the birooted image of tree $t$. By extension, for every set $X$ of $F$-trees, the language $L_{X}=$ $\{\langle t, 1\rangle \in \mathcal{B}(F): t \in X\}$ of birooted tree images of trees of $X$ is called the birooted tree image of the language $X$.

Theorem 3. For every regular language $X$ of complete finite $F$-trees, we have $L_{X}=U_{X} \cap D_{X}$ for some regular language $U_{X}$ of birooted $F$-trees and the complement $D_{X}$ of some regular language $\mathcal{B}(F)-D_{X}$ of birooted $F$-trees. 
Proof. This essentially follows from Theorem 2. More precisely, let $X$ be a regular language of finite $F$-tree.

We observe first that for every complete $F$-tree $t_{1}$ and $t_{2}$, their birooted images $\left\langle t_{1}, 1\right\rangle$ and $\left\langle t_{2}, 1\right\rangle$ are incomparable in the natural order. It follows that the element of $L_{X}$ form an anti-chain in the natural order. It follows that, given $U_{x}=\left\{y \in \mathcal{B}(F): \exists x \in L_{X}, x \leq y\right\}$ the upward closure of $L_{X}$ and $D_{X}=\{y \in$ $\left.\mathcal{B}(F): \exists x \in L_{X}, y \leq x\right\}$ the downward closure of $L_{X}$, we have $L_{X}=U_{X} \cap L_{X}$. We conclude the proof by observing that if $X$ is regular then it is definable in MSO. This implies that the languages $L_{X}, D_{X}$ and $U_{X}$ are also definable in MSO. We conclude by applying Theorem 2, that ensures that both $U_{X}$ and $\mathcal{B}(X)-D_{X}$ are regular languages of birooted trees.

\section{Quasi-recognizable languages of birooted $F$-trees}

Intimately related to the theory of non-regular semigroups initiated by Fountain in the 70s (see e.g [4]), the notion of recognizability by premorphisms is proposed in [6] (generalized in [9]) to define languages of positive (resp. arbitrary) overlapping tiles. This notion is extended here to languages of birooted $F$-trees.

\subsection{Definition}

Let $S$ be a monoid partially ordered by a relation $\leq_{S}$ (or just $\leq$ when there is no ambiguity). We always assume that the order relation $\leq$ is stable under product, i.e. if $x \leq y$ then $x z \leq y z$ and $z x \leq z y$ for every $x, y$ and $z \in S$. The set $U(S)$ of subunits of the partially ordered monoid $S$ is defined by $U(S)=\{y \in S: y \leq 1\}$.

A partially ordered monoid $S$ is an adequately ordered monoid when all subunits of $S$ are idempotents, and for every $x \in S$, both the minimum of right local units $x^{L}=\min \{y \in U(S): x y=x\}$ and the minimum of left local units $x^{R}=\min \{y \in U(S): y x=x\}$ exist and belong to $U(S)$. The subunits $x^{L}$ and $x^{R}$ are respectively called the left projection and the right projection of $x$.

Since subunits are assumed to be idempotents, one can check that they commute and thus, ordered by the monoid order, form a meet semilattice with the product as the meet operator. It follows that when $x$ is itself a subunit, we have $x=x^{L}=x^{R}$. In other words, in an adequately ordered monoid, both left and right projection mappings are indeed projections from $S$ onto $U(S)$.

Examples. Every monoid $S$ extended with the trivial order $x \leq y$ when $x=y$ is a adequately ordered monoid with $x^{L}=x^{R}=1$ for every $x \in S$. These adequately ordered monoids are called trivial.

Every inverse monoid $S$ ordered by the natural order is an adequately ordered monoid with $x^{L}=x^{-1} x$ and $x^{R}=x x^{-1}$ for every $x \in S$.

As a particular case, the monoid $\mathcal{B}^{1}(F)$ ordered by the natural order is also an adequately ordered monoid. The subunits of $\mathcal{B}^{1}(F)$ are, when distinct from 
0 or 1 , the birooted $F$-trees of the form $\langle t, 1\rangle$ and, indeed, for every birooted $F$-tree $B=\langle t, u\rangle$ we have $B^{R}=\langle t, 1\rangle$ and $B^{L}=\left\langle t_{u}, 1\right\rangle$.

For every set $Q$, the relation monoid $\mathcal{P}(Q \times Q)$ ordered by inclusion is also an adequately ordered monoid with, for every $X \subseteq Q \times Q, X^{L}=\{(q, q) \in Q \times Q$ : $(p, q) \in X\}$ and $X^{R}=\{(p, p) \in Q \times Q:(p, q) \in X\}$.

A mapping $\theta: S \rightarrow T$ between two adequately ordered monoids is a premorphism when $\theta(1)=1$ and, for every $x$ and $y \in S$, we have $\theta(x y) \leq_{T} \theta(x) \theta(y)$ and if $x \leq_{S} y$ then $\theta(x) \leq_{T} \theta(y)$. A premorphism $\theta: S \rightarrow T$ is an adequate premorphism when for every $x$ and $y \in S$ we have $\theta\left(x^{L}\right)=(\theta(x))^{L}, \theta\left(y^{R}\right)=(\theta(y))^{R}$ and, if $x y \neq 0$ with $x^{L} \vee y^{R}=z \prec 1$, i.e. the product $x y$ is a disjoint product, then $\theta(x y)=\theta(x) \theta(y)$.

A language $L \subseteq \mathcal{B}(F)$ of birooted tree is a quasi-recognizable language when there exists a finite adequately ordered monoid $S$ and an adequate premorphism $\theta: \mathcal{B}(F) \rightarrow S$ such that $L=\theta^{-1}(\theta(L))$.

Theorem 4. Let $\theta: F I M(A) \rightarrow S$ be an adequate premorphism with finite $S$. For every $B \in \mathcal{B}(F)$ the image $\theta(B)$ of the birooted $F$-tree $B$ by the adequate premorphism $\theta$ is uniquely determined by the structure of $B$, the structure of $S$ and the image by $\theta$ of elementary birooted $F$-trees.

Proof. This essentially follows from the adequacy assumption and the strong decomposition property (Lemma 1).

\subsection{From birooted tree automata to quasi-recognizable languages}

Now we want to show that every finite state birooted automaton induces an adequate premorphism that recognizes the same language.

Theorem 5. Let $L \subseteq \mathcal{B}(F)$ be a language of birooted $F$-trees. If $L$ is recognizable by a finite state birooted tree automaton then it is recognizable by an adequate premorphism into a finite adequately ordered monoid.

Proof. Let $L \subseteq \mathcal{B}(F)$ and let $\mathcal{A}=\langle Q, \delta, \Delta, T\rangle$ be a finite birooted tree automaton such that $L=L(\mathcal{A})$.

We define the mapping $\varphi_{\mathcal{A}}: \mathcal{B}(F) \rightarrow \mathcal{P}(Q \times Q)$ by saying that $\varphi_{\mathcal{A}}(B)$ is, for every birooted $F$-tree $B=\langle t, u\rangle \in \mathcal{B}(F)$, the set of all pairs of state $(p, q) \in Q \times Q$ such that there exists a run $\rho: \operatorname{dom}(t) \rightarrow Q$ such that $p=\rho(1)$ and $q=\rho(u)$. The mapping $\varphi_{\mathcal{A}}$ is extended to 0 by taking $\varphi_{\mathcal{A}}(0)=\emptyset$ and, to 1 by taking $\varphi(1)=I_{Q}=\{(q, q) \in Q \times Q: q \in Q\}$.

The fact $\mathcal{P}(Q \times Q)$ is an adequately ordered monoid have already been detailled in the examples above. By definition we have $L=\varphi^{-1}(\mathcal{X})$ with $\mathcal{X}=$ $\{X \subseteq Q \times Q: X \cap T \neq 0\}$. Then, we prove that $\varphi_{\mathcal{A}}$ is indeed an adequate premorphism.

More precisely, the fact $\varphi_{\mathcal{A}}$ is monotonic is immediate. Indeed, for every birooted $F$-tree $\langle s, u\rangle$ and $\langle t, v\rangle$, if $\langle s, u\rangle \leq\langle t, v\rangle$ this means that $u=v$ thus, for 
every run $\rho: \operatorname{dom}(s) \rightarrow Q$ of $\mathcal{A}$ on $\langle s, u\rangle$, the mapping $\rho$ restricted to $\operatorname{dom}(t)$ is clearly a run of $\mathcal{A}$ on $\langle s \mid \operatorname{dom}(t), u\rangle=\langle t, u\rangle$.

Left and right projections preservation immediately follows from their characterizations in both $\mathcal{B}(F)$ and $\mathcal{P}(Q \times Q)$ and the definition of $\varphi_{\mathcal{A}}$.

Now we show that $\varphi_{\mathcal{A}}$ is submultiplicative. Let $\langle s, u\rangle$ and $\langle t, v\rangle$ be two birooted trees. In the case $\langle s, u\rangle \cdot\langle t, v\rangle=0$ we are done, since $\varphi_{\mathcal{A}}(0)=\emptyset$. Otherwise, let $\rho$ be a run of $\mathcal{A}$ on the product $\langle s, u\rangle \cdot\langle t, v\rangle$. By definition of the product, the mapping $\rho_{1}: \operatorname{dom}(s) \rightarrow Q$ defined by $\rho_{1}(w)=\rho(w)$ for every $w \in \operatorname{dom}(s)$ is clearly a run of $\mathcal{A}$ on $\langle s, u\rangle$. Similarly, the run $\rho_{2}: \operatorname{dom}(t) \rightarrow Q$ defined by $\rho_{2}(w)=\rho(u w)$ for every $w \in \operatorname{dom}(s)$ is also a run of $\mathcal{A}$ on $\langle t, v\rangle$. Now, since $\rho_{1}(u)=\rho_{2}(1)$ and that construction applies for every run $\rho$, this shows that $\varphi_{\mathcal{A}}(\langle s, u\rangle \cdot\langle t, v\rangle) \subseteq \varphi_{\mathcal{A}}(\langle s, u\rangle) \cdot \varphi_{\mathcal{A}}(\langle t, v\rangle)$.

Last, it remains to show that $\varphi_{\mathcal{A}}$ preserves disjoint products. Assume that the product $\langle s, u\rangle \cdot\langle t, v\rangle$ is disjoint. This means that $s(u)=t(1)$ and $\operatorname{dom}(s) \cap$ $u \cdot \operatorname{dom}(t)=\{u\}$.

Let $(p, q) \in \varphi_{\mathcal{A}}(\langle s, u\rangle) \cdot \varphi_{\mathcal{A}}(\langle t, v\rangle)$. By definition of the product of relations, this means that there exists $q^{\prime} \in Q$, such that $\left(p, q^{\prime}\right) \in \varphi_{\mathcal{A}}(\langle s, u\rangle)$ and $\left(q^{\prime}, q\right) \in$ $\varphi_{\mathcal{A}}(\langle t, v\rangle)$. But then, by definition of $\varphi_{\mathcal{A}}$ this means that there exists a run $\rho_{1}: \operatorname{dom}(s) \rightarrow Q$ of $\mathcal{A}$ on $\langle s, u\rangle$ and a run $\rho_{2}: \operatorname{dom}(t) \rightarrow Q$ of $\mathcal{A}$ on $\langle t, v\rangle$ such that $\rho_{1}(1)=p, \rho_{1}(u)=q^{\prime}, \rho_{2}(1)=q^{\prime}$ and $\rho_{2}(v)=q$.

Let then $\rho: \operatorname{dom}(s) \cup u \cdot \operatorname{dom}(t): \rightarrow Q$ defined by $\rho(w)=\rho_{1}(w)$ for every $w \in \operatorname{dom}(s)$, and $\rho(u w)=\rho_{2}(w)$ for every $w \in \operatorname{dom}(t)$. Since the product of the two birooted $F$-trees is a disjoint product, we have $\operatorname{dom}(s) \cap u \cdot \operatorname{dom}(t)=\{u\}$ with $\rho_{1}(u)=q^{\prime}=\rho_{2}(1)$ hence $\rho$ is well defined. As it is clearly a run of $\mathcal{A}$ on the (non zero) product $\langle s, u\rangle \cdot\langle t, v\rangle$ with $\rho(1)=p$ and $\rho(u v)=q$, this means we indeed have $(p, q) \in \varphi_{\mathcal{A}}(\langle s, u\rangle \cdot\langle t, v\rangle)$.

As this holds for arbitrary pair of states $(p, q) \in \varphi_{\mathcal{A}}(\langle s, u\rangle) \cdot \varphi_{\mathcal{A}}(\langle t, v\rangle)$ this proves that $\varphi_{\mathcal{A}}(\langle s, u\rangle) \cdot \varphi_{\mathcal{A}}(\langle t, v\rangle) \subseteq \varphi_{\mathcal{A}}(\langle s, u\rangle \cdot\langle t, v\rangle)$ and thus concludes the proof.

\subsection{From quasi-recognizability to MSO}

The following theorem tells how quasi-recognizability and MSO definability are related.

Theorem 6. Let $\theta: F I M(A) \rightarrow S$ be an adequate premorphism with finite $S$. For every $X \subseteq S$, the language $\theta^{-1}(X)$ is definable in Monadic Second Order Logic.

Proof. Let $\theta: F I M(A) \rightarrow S$ as above and let $X \subseteq S$. Uniformly computing the value of $\theta$ on every birooted tree by means of an MSO formula is done by adapting Shelah's decomposition techniques [17]. More precisely, we show that the strong decomposition provided by Lemma 1 is indeed definable in MSO. Then, the computation of the value of $\theta$ on every birooted rooted $B$ can be 
done from the value of $\theta$ on the elementary birooted trees and the sub-birooted $F$-trees that occur in such a decomposition.

More precisely, we first show that the predecessor relation $\prec_{p}$ (and thus, by transitive closure, the prefix relation $\leq_{p}$ as well) is definable in MSO. This amounts to saying that there exists an MSO formula $\varphi_{p}(x, y)$ such that, for every birooted tree $\langle t, u\rangle$, for every vertex $v$ and $w \in \operatorname{dom}(t)$, we have $\langle t, u\rangle \models \varphi_{p}(v, w)$ if and only if $v \prec_{p} w$.

Indeed, defining $\varphi_{p}(x, y)$ amounts to saying that there exists a partition of $\operatorname{dom}(t)$ in three sets of vertices $X_{0}, X_{1}$ and $X_{2}$ such that the (input) root 1 belongs to $X_{0}$, all its neighbors (or immediate successors) belong to $X_{1}$, and for every vertex $z \in Z$ distinct from the input root, given $i \in\{0,1,2\}$ such that $z \in X_{i}$, given $j=i-1 \bmod 3$ and $k=i+1 \bmod 3$, the vertex $z$ has a single neighbor in $X_{j}$ (the unique predecessor of $z$ in the predecessor relation $\prec_{p}$ ) and all other neighbors of $z$ belong to $X_{k}$ (the successors of $z$ in the predecessor relation $\prec_{p}$ ).

As a consequence, since the reflexive and transitive closure of a definable binary relation is also definable in MSO, there exists a formula $\varphi_{p}^{*}(x, y)$ such that $\langle t, u\rangle \models \varphi_{p}^{*}(v, w)$ if and only $v \leq_{p} w$.

This also means that for every birooted tree $B=\langle t, u\rangle$, the set $U=\{z \in$ $\left.\operatorname{dom}(t): 1 \leq_{p} z \leq_{p} u\right\}$ is also MSO definable in every birooted tree $\langle t, u\rangle$ and, as well, for every vertex $v \in \operatorname{dom}(t)$, the sub-birooted tree $B_{v}^{p}$. Here, by saying the birooted $F$-tree $B_{v}^{p}$ is definable in MSO we mean that its domain $D^{p}(v)$ (defined in the proof of Lemma 1) is definable and thus its structure: the vertex labels and the edge relations, is just obtained by restricting those of $B$ to the domain $D^{p}(v)$.

The next step is then the following. Given a finite collection of set variables $\left\{Y_{s}\right\}_{s \in U(S)}$, one variable $Y_{s}$ per element $s \in U(S)$, writing $\bar{Y}$ for the tuple of such variables, we claim that there exists a formula $\varphi(\bar{Y})$ such that for every birooted $F$-tree $\langle t, u\rangle$ for every $v \in \operatorname{dom}(t)$, for every subunit $s \in U(S)$, we have $\langle t, u\rangle \models \exists \bar{Y}\left(v \in Y_{s} \wedge \varphi(\bar{Y})\right)$ if and only if $\theta\left(B_{v}^{p}\right)=s$.

Indeed, this amounts to saying that $\left\{Y_{s}\right\}_{s \in U(S)}$ form a partition (with possible empty sets) of $\operatorname{dom}(t)$ such that, for every vertex $v \in \operatorname{dom}(t)$, if $v$ is a leaf w.r.t. the prefix order $\leq_{p}$ then $s=\theta\left(B_{v}^{p}\right)=\theta\left(B_{t(v)}\right)$ and we check that $v$ belongs to $Y_{s}$ or, if $v$ is not a leaf, then we must have $v \in X_{s}$ with, by adequacy assumption on $\theta$, the value of $s$ that is uniquely determined by

$$
s=\prod\left\{\left(\theta\left(B_{v, w}^{p}\right) \cdot s_{w}\right)^{R}: v \prec_{p} w\right\}
$$

with $s_{w} \in S$ is the unique element of $S$ such that $w \in Y_{s_{w}}$. Indeed, by the proof of Lemma 1, we known that $B_{v}^{p}=\prod\left\{\left(B_{v, w}^{p} \cdot B_{w}^{p}\right)^{R}: w \in \operatorname{dom}(r), v \prec_{p} w\right\}$ with disjoint products only and the adequacy assumption applies. As the product is of a bounded size, we can check that $v \in Y_{s}$.

Then, for every birooted tree $B=\langle t, u\rangle$, given the ordered prefixes of $u$ described by $u_{0}=1<_{p} u_{1}<_{p} u_{2}<_{p} \cdots<_{p} u_{n-1}<_{p} u_{n}=u$ the value of $\theta(B)$ 
can be computed as the element $s \in S$ defined by

$$
s=\theta\left(B_{u_{0}}^{p}\right) \theta\left(B_{u_{0}, u_{1}}^{p}\right) \theta\left(B_{u_{1}}^{p}\right) \cdots \theta\left(B_{u_{n-1}}^{p}\right) \theta\left(B_{u_{n-1}, u_{n}}^{p}\right) \theta\left(B_{u_{n}}^{p}\right)
$$

Indeed, by Lemma 1 we have $B=B_{u_{0}}^{p} B_{u_{0}, u_{1}}^{p} B_{u_{1}}^{p} \cdots B_{u_{n-1}}^{p} B_{u_{n-1}, u_{n}}^{p} B_{u_{n}}^{p}$ with disjoint products only hence, by adequacy of $\theta$, the claim. As all these values are computable, either as image by $\theta$ of elementary birooted trees, or, by induction, by observing that for every prefix $u^{\prime}$ of $u$ we have $\theta\left(B_{u^{\prime}}^{p}\right)=s^{\prime}$ if and only if $u^{\prime} \in Y_{s^{\prime}}$. Then, checking that $v \in Y_{s}$ by "computing" in MSO the value $s$ can be done, say, by a left to right "traversal" of the path from 1 to $u$, simulating the underlying finite state word automaton induced by $S$ on the (images of) elementary birooted trees. This concludes the proof.

\subsection{Quasi-recognizable languages vs MSO definable languages}

For the picture to be complete, it remains to characterize the class of quasirecognizable languages w.r.t. the class of languages definable in Monadic Second Order Logic.

Theorem 7. Let $L \subseteq \mathcal{B}(F)$ be a language of birooted $F$-trees. The following properties are equivalent:

(1) the language $L$ is quasi-recognizable,

(2) the language $L$ is a finite boolean combination of upward closed MSO definable languages,

(3) the language $L$ is a finite boolean combination of languages recognized by finite state birooted tree automata.

Proof. The fact that (1) implies (2) essentially follows from Theorem 6 . The fact (2) implies (3) immediately follows from Theorem 2. Last, we prove, by classical argument (e.g. cartesian product of monoids) that the class of quasi-recognizable languages is closed under boolean operations. Then, by applying Theorem 5 this proves that (3) implies (1).

More precisely, Let $L \subseteq \mathcal{B}(F)$ be a language of birooted $F$-trees.

(1) implies (2). We assume that $L$ is recognized by some adequate premorphism $\theta: \mathcal{B}(F) \rightarrow S$. By definition, we have $L=\theta^{-1}(\theta(L))$ hence

$$
\theta^{-1}(\theta(L))=\bigcup_{x \in \theta(L)} \theta^{-1}\left(D_{x}\right) \cap \theta^{-1}\left(U_{x}\right)
$$

with $U_{x}=\{y \in S: x \leq y\}$ and $D_{x}=\{y \in S: y \leq x\}$ for every $x \in S$. Indeed, for every $x \in S$, we have $\theta^{-1}(x)=\theta^{-1}\left(U_{x}\right) \cap \theta^{-1}\left(D_{x}\right)$. The inclusion $\theta^{-1}(x) \subseteq$ $\theta^{-1}\left(U_{x}\right) \cap \theta^{-1}\left(D_{x}\right)$ is immediate. Conversely, let $B \in \theta^{-1}\left(U_{x}\right) \cap \theta^{-1}\left(D_{x}\right)$. Since $B \in \theta^{-1}\left(U_{x}\right)$ we have $x \leq \theta(B)$ and since $B \in \theta^{-1}\left(D_{x}\right)$ we have $\theta(B) \leq x$ hence $\theta(B)=x$ and thus $B \in \theta^{-1}(x)$.

We prove (2) by observing that both $\theta^{-1}\left(U_{x}\right)$ and $\overline{\theta^{-1}\left(D_{x}\right)}=\theta^{-1}\left(S-D_{x}\right)$ are upward closed (and recognized by $\theta$ ) hence, by Theorem 6 , they are MSO definable. 
(2) implies (3). This immediately follows from Theorem 2 that ensures that every upward closed and MSO definable languages is recognized by a finite state birooted tree automaton.

(3) implies (1). Assume that $L$ is a finite boolean combination of languages recognized by birooted tree automata. We want to show that $L$ is quasi-recognizable.

By Theorem 5, every such a regular language is quasi-recognizable. Since the class of quasi-recognizable languages is obviously closed under complement it suffices to prove that it is closed under intersection.

But this is easily done using classical algebraic tools on monoids [15]. Indeed, given two adequate premorphisms $\theta_{1}: \mathcal{B}(F) \rightarrow S_{1}$ and $\theta_{2}: \mathcal{B}(F) \rightarrow S_{2}$, the mapping $\theta: \mathcal{B}(F) \rightarrow S_{1} \times S_{2}$ defined by $\varphi(B)=\left(\varphi_{1}(B), \varphi_{2}(B)\right)$ is an adequate premorphism in the product monoid $S_{1} \times S_{2}$ ordered by the product order. Then, for every $X \subseteq S_{1}$ and $Y \subseteq S_{2}$ we have $\varphi_{1}^{-1}(X) \cap \varphi_{2}^{-1}(Y)=\varphi^{-1}(X \times Y)$. This concludes the proof.

Corollary 8. The birooted image of every regular languages of F-tree is recognizable by an adequate premorphism in a finite adequately ordered monoid.

Proof. This follows from Theorem 3 and Theorem 7.

\section{Conclusion}

Studying languages of birooted $F$-trees, structures that generalize $F$-terms, we have thus defined a notion of automata, a related notion of quasi-recognizability and we have characterized quite in depth their expressive power in relationship with language definability in Monadic Second Order Logic.

As a particular case, our results provide a new algebraic characterization of the regular languages of finite $F$-trees. Potential links with the preclones approach [5] or the forest algebra approach [3,2] need to be investigated further.

\section{References}

1. A. Blumensath. Recognisability for algebras of infinite trees. Theor. Comput. Sci., 412(29):3463-3486, 2011.

2. M. Bojanczyk, H. Straubing, and I. Walukiewicz. Wreath products of forest algebras, with applications to tree logics. Logical Methods in Computer Science, 8(3), 2012.

3. M. Bojańczyk and I. Walukiewicz. Forest algebras. In Logic and Automata, pages 107-132, 2008.

4. C. Cornock and V. Gould. Proper two-sided restriction semigroups and partial actions. Journal of Pure and Applied Algebra, 216:935-949, 2012.

5. Z. Ésik and P. Weil. On logically defined recognizable tree languages. In Found. of Soft. Techno. and Theoretical Computer Science (FSTTCS), pages 195-207, 2003. 
6. D. Janin. Quasi-recognizable vs MSO definable languages of one-dimensional overlaping tiles. In Mathematical Foundations of Computer Science (MFCS), volume 7464 of $L N C S$, pages $516-528,2012$.

7. D. Janin. Walking automata in the free inverse monoid. Technical Report RR1464-12 (revised april 2013), LaBRI, Université de Bordeaux, 2012.

8. D. Janin. On languages of one-dimensional overlapping tiles. In International Conference on Current Trends in Theory and Practice of Computer Science (SOFSEM), volume 7741 of $L N C S$, pages 244-256, 2013.

9. D. Janin. Overlaping tile automata. In 8th International Computer Science Symposium in Russia (CSR), volume 7913 of LNCS, pages 431-443. Springer, 2013.

10. J. Kellendonk and Mark V. Lawson. Tiling semigroups. Journal of Algebra, 224(1):140 - 150, 2000.

11. M. V. Lawson. McAlister semigroups. Journal of Algebra, 202(1):276 - 294, 1998.

12. D. Perrin and J.-E. Pin. Semigroups and automata on infinite words. In J. Fountain, editor, Semigroups, Formal Languages and Groups, NATO Advanced Study Institute, pages 49-72. Kluwer academic, 1995.

13. J.-E. Pin. Relational morphisms, transductions and operations on languages. In Formal Properties of Finite Automata and Applications, volume 386 of LNCS, pages 34-55. Springer, 1989.

14. J.-E. Pin. Finite semigroups and recognizable languages: an introduction. In J. Fountain, editor, Semigroups, Formal Languages and Groups, NATO Advanced Study Institute, pages 1-32. Kluwer academic, 1995.

15. J-.E. Pin. Chap. 10. Syntactic semigroups. In Handbook of formal languages, Vol. I, pages 679-746. Springer Verlag, 1997.

16. H. E. Scheiblich. Free inverse semigroups. Semigroup Forum, 4:351-359, 1972.

17. S. Shelah. The monadic theory of order. Annals of Mathematics, 102:379-419, 1975.

18. W. Thomas. Chap. 7. Languages, automata, and logic. In Handbook of Formal Languages, Vol. III, pages 389-455. Springer Verlag, 1997.

19. T. Wilke. An algebraic theory for regular languages of finite and infinite words. Int. J. Alg. Comput, 3:447-489, 1993. 